\title{
Modelo Fuzzy Takagi-Sugeno para controle do ângulo de articulação do joelho de pacientes paraplégicos
}

\author{
Takagi-Sugeno fuzzy model for knee joint angle control of \\ paraplegic patients
}

\author{
Dayanne Nayara Telles de Proença Diogo ${ }^{1}$; Luiz Henrique Ravagnani Bueno² ; \\ Márcio Roberto Covacic ${ }^{3}$; Ruberlei Gaino ${ }^{4}$.
}

\section{Resumo}

Neste trabalho foi elaborado o controle em malha fechada, por meio de estimulação elétrica funcional, para o movimento da perna de um paciente paraplégico. Isto é feito utilizando modelos fuzzy Takagi-Sugeno, os quais resolvem classes de sistemas não lineares. O problema é descrito por desigualdades matriciais lineares (LMI) atendendo a especificações, tais como estabilidade segundo o teorema de Lyapunov, taxa de decaimento e restrição no sinal de entrada. Este controle foi projetado para variar o ângulo de articulação do joelho na faixa de $0^{\circ}$ a $45^{\circ}$, mediante a estimulação elétrica no músculo quadríceps. $\mathrm{O}$ sinal de realimentação é relacionado ao ângulo de articulação do joelho $(\theta v)$, estabilizando-o em $45^{\circ}$. Palavras-chave: Estimulação elétrica funcional. Modelos fuzzy Takagi-Sugeno. LMI. Sistema não linear. Lyapunov.

\begin{abstract}
In this paper, a feedback control has been developed, using functional electrical stimulation in order to control the movement of the leg of a paraplegic patient. The control design uses Takagi-Sugeno fuzzy models, which solves classes of nonlinear systems. The problem is described by linear matrix inequalities (LMI), reaching the specifications of stability according to Lyapunov theorem, decay rate and restriction on the input signal. The control system was designed to vary the knee joint angle from $0^{\circ}$ to $45^{\circ}$ by electrical stimulation of the quadriceps muscle. The feedback signal is related to the knee joint angle $(\theta v)$, stabilizing it at $45^{\circ}$.
\end{abstract}

Keywords: Functional electrical stimulation. Takagi-Sugeno fuzzy models. LMI. Nonlinear system. Lyapunov.

\footnotetext{
${ }^{1}$ Estudante de graduação do Departamento de Engenharia Elétrica, Universidade Estadual de Londrina - dnproenca@gmail.com

2 Estudante de graduação do Departamento de Engenharia Elétrica, Universidade Estadual de Londrina - luizhrbueno@gmail.com

3 Docente do Departamento de Engenharia Elétrica da Universidade Estadual de Londrina - DEEL-UEL; marciocovacic@gmail. com

4 Docente do Departamento de Engenharia Elétrica da Universidade Estadual de Londrina - DEEL-UEL; ruberlei.gaino@gmail. com.
} 


\section{Introdução}

A Estimulação Elétrica Funcional (Functional Electrical Stimulation - FES) tem sido utilizada na restauração de movimentos em pacientes paraplégicos, tetraplégicos e hemiplégicos. Estudos têm sido realizados com o objetivo do controle do movimento de pacientes paraplégicos utilizando FES e diferentes modelos matemáticos de músculos e da articulação do joelho (FERRARIN; PEDOTTI, 2000).

A motivação para a realização deste trabalho concentra-se no grande potencial que a área possui em desenvolver-se. No Congresso Brasileiro de Engenharia Biomédica (CBEB) de 2008, houve dois trabalhos publicados sobre reabilitação de pacientes paraplégicos com FES em malha fechada, realizados por (GAINO et al., 2008) e (PRADO et al., 2008). Estes trabalhos abordam a solução dos problemas vivenciados por pacientes paraplégicos e hemiplégicos com o objetivo de melhorar a qualidade de suas vidas.

Na Figura 1, adaptada de (GAINO et al., 2007a), ilustra-se o diagrama geral do sistema de controle. No bloco "Estação de Controle", pretende-se utilizar os códigos de controle embarcado em estudo, sistemas não lineares como modelos fuzzy TakagiSugeno (TEIXEIRA et al., 2006), (GAINO, 2009), (GAINO et al., 2011), (COVACIC et al., 2012) e sistemas lineares (GAINO et al., 2008), (PRADO et al., 2008) , (SANCHES et al., 2010).

Figura 1 - Ilustração do Hardware utilizado para controle.

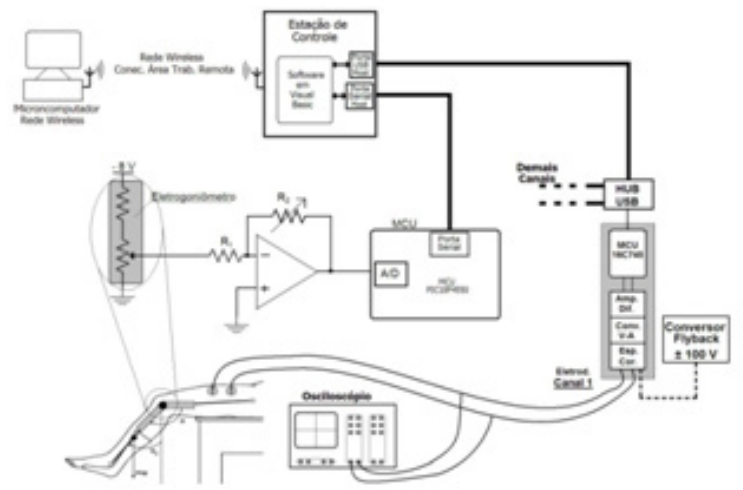

Fonte: (SILVA, 2007).
Figura 2 - Estimulação elétrica do nervo por meio de eletrodos externos.

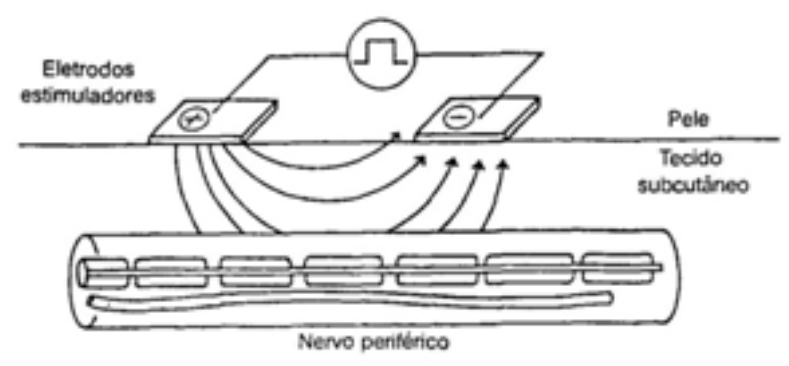

Fonte: (ROBINSON, 2001).

Quando, em alguns instantes, a corrente se dá através da membrana celular, ela provoca a despolarização da membrana, que dependendo de sua intensidade, é suficiente para provocar um Potencial de Ação (PA), que se propagará através da membrana. Esse PA é idêntico ao produzido pelo SNC, porém se propaga nas duas direções da fibra nervosa.

A amplitude/intensidade e a duração do estímulo elétrico devem ter um valor mínimo para evocar um PA, isto é, devem estar acima de um limiar. A proximidade do eletrodo com a fibra nervosa, seu diâmetro, entre outras características, determinam a velocidade de recrutamento, bem com o número de fibras recrutadas (ROBINSON, 2001).

Estudos do neuroestimulador com FES em malha fechada com aplicação em pacientes paraplégicos foram realizados em (GAINO et al., 2007b).

Em (TEIXEIRA et al., 2006), foram realizados pela primeira vez estudos e simulações da posição da perna de um paciente paraplégico. Com o uso de modelos fuzzy Takagi-Sugeno para controlar a posição da perna do paciente paraplégico projetando um regulador de estados para o ângulo de articulação do joelho em torno de $30^{\circ}$, com retroalimentação, consideram-se todas as variáveis de estado disponíveis, utilizando como sensor o eletrogoniômetro.

Uma variação deste modelo foi publicada em (GAINO et al., 2011). O projeto do regulador foi realizado para o mesmo ponto. Considerando que não 
estava acessível o estado da posição angular, a partir da aceleração angular, obteve-se uma estimativa da velocidade angular, usando o acelerômetro.

No presente trabalho, o controlador foi projetado visando variar o ângulo da articulação do joelho no intervalo de $0^{\circ}$ a $45^{\circ}$, mediante a estimulação elétrica no músculo quadríceps. No sistema de controle foram projetados em simulação, controladores com Modelos fuzzy Takagi-Sugeno, que resolvem classes de sistemas não lineares, aplicados ao caso do paciente paraplégico (TAKAGI; SUGENO, 1985), (TEIXEIRA; ZAK, 1999).

Utilizando técnicas de controle não linear por meio dos modelos fuzzy Takagi-Sugeno (T-S) e desigualdades matriciais lineares (Linear Matrix Inequalities - LMI) consegue-se atingir a estabilidade em malha fechada que possibilita a otimização da largura de pulso, diminuindo a fadiga do músculo, tornando o processo controlável e mais eficiente (REINER, 1998). Atende-se assim às especificações da estabilidade segundo Lyapunov, taxa de decaimento e restrição de entrada, aperfeiçoando o controle.

O modelo global do sistema é obtido através da combinação fuzzy destes modelos lineares locais. Para cada modelo linear local é considerado um controle de realimentação linear. $O$ regulador global resultante, que é não linear, em geral, é uma combinação fuzzy de cada regulador linear individual (TANAKA et al., 1998).

Nas simulações realizadas em MATLAB, os sinais disponíveis para o projeto de controle são representados pelas variáveis de estado da planta, que são o deslocamento angular, a velocidade angular e o torque ativo produzido pela estimulação elétrica. O sinal de realimentação se relaciona com o ângulo da articulação do joelho, alterando, assim, a largura de pulso (TEIXEIRA et al., 2006), (GAINO, 2009), (GAINO, 2011).

As LMIs são conhecidas e usadas largamente como mostrado em (TANAKA; WANG, 2001). Para este caso, leis menos conservativas para a estabilidade funcionaram adequadamente e a estabilidade encontra-se sem maiores problemas. Casos com relaxamento das LMIs para encontrar a estabilidade também poderiam ter sido utilizados (TEIXEIRA et al., 2003).

No estudo de (GAINO, 2009), todos os modelos fuzzy tem a função de pertinência calculada pelo método de (TANIGUCHI et al., 2001), na qual essa é escrita em função da não linearidade em $f_{21}\left(x_{1}(\mathrm{t})\right)$, localizada na matriz $\mathrm{A}_{\mathrm{nxn}}$, sendo $n$ é a dimensão da matriz.

Neste artigo, o projeto com modelos fuzzy Takagi-Sugeno considera a função de pertinência do tipo gaussiana proposta em (PIETROBOM, 1999). Optou-se pelo uso dessa função devido à praticidade da identificação por meio do processo descrito em (TAKAGI; SUGENO, 1985).

Um estudo em simulação foi realizado por (BIAZETO, 2011), com base nas equações do modelo dinâmico da posição da perna do paciente paraplégico, utilizando a técnica recursiva (RLSRecursive Least Squares), e a função de pertinência gaussiana, na identificação do sistema para projeto de controle.

Em (COVACIC et al, 2012), os revisores evidenciaram o uso da técnica de controle com estrutura variável na dinâmica do paciente paraplégico.

\section{Modelo dinâmico da posição da perna do paciente paraplégico}

$\mathrm{Na}$ modelagem proposta por (FERRARIN; PEDOTTI, 2000), relaciona-se a largura do pulso aplicado com o torque gerado em torno da articulação do joelho, considerando o membro inferior de uma cadeia cinemática aberta composta pelos segmentos rígidos da coxa e do complexo canela-pé, como mostra a Figura 3. 
Figura 3 - Sistema de controle - complexo canela-pé.

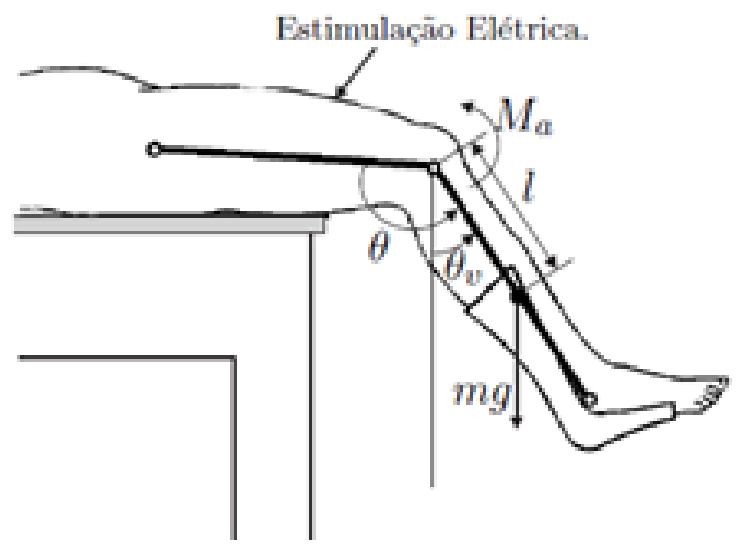

Fonte: adaptado de (FERRARIN; PEDOTTI, 2000).

No modelo, $\theta, \quad \theta v$ e $M_{a 0}$ representam, respectivamente, o ângulo do joelho (ângulo entre a canela e a coxa no plano sagital), o ângulo da canela (ângulo entre a canela e o eixo vertical no plano sagital) e o torque ativo produzido pela estimulação elétrica $(\mathrm{P})$.

A equação de estados não lineares proposta em (TEIXEIRA et al., 2006), (GAINO, 2009), (GAINO et al., 2011) e (COVACIC et al., 2012), que representa o movimento da articulação do joelho ao estimulo elétrico aplicado, é dada por:

$\left[\begin{array}{l}\dot{x}_{1}(t) \\ \dot{x}_{2}(t) \\ \dot{x}_{3}(t)\end{array}\right]=\left[\begin{array}{ccc}0 & 1 & 0 \\ \tilde{f}_{21}\left(x_{1}(t)\right) & \frac{-B}{J} & \frac{1}{J} \\ 0 & 0 & \frac{-1}{\tau}\end{array}\right]\left[\begin{array}{l}x_{1}(t) \\ x_{2}(t) \\ x_{3}(t)\end{array}\right]+\left[\begin{array}{l}0 \\ 0 \\ \frac{G}{\tau}\end{array}\right] P_{N}$

sendo $\quad x_{1}=\Delta \theta_{v}=\theta_{v}-\theta_{v 0}, \quad x_{2}=\dot{x}_{1}=\Delta \dot{\theta}_{v} \quad \mathrm{e}$ $x_{3}=\Delta M_{a}=M_{a}-M_{a 0}$. O ponto de operação do sistema é $\left(\theta_{v}, \dot{\theta}_{v}, M_{a}\right)=\left(\theta_{v}, 0, M_{a 0}\right)$. A função $f_{21}\left(\mathrm{x}_{1}(\mathrm{t})\right)$ é uma não linearidade do sistema, descrita por:

$$
\begin{gathered}
\tilde{f}_{21}\left(x_{1}(t)\right)=\frac{1}{J x_{1}}\left(-m g l \sin \left(\Delta \theta_{v}+\theta_{v 0}\right)-\right. \\
\lambda e^{-E\left(\Delta \theta_{v}+\theta_{v 0}\right)} e^{-E \frac{\pi}{2}}\left(\Delta \theta_{v}+\theta_{v 0}+\frac{\pi}{2}-\omega\right)+ \\
\left.M_{a 0}\right) \\
\mathrm{P}_{0}=\frac{M_{a 0}}{G} \\
M_{a 0}=m g l \sin \left(\theta_{v}\right)+\lambda e^{-E \theta_{v}} e^{-E \frac{\pi}{2}}\left(\theta_{v}+\frac{\pi}{2}-\omega\right)
\end{gathered}
$$

Considerado as seguintes medidas antropométricas (FERRARIN; PEDOTTI, 2000), os parâmetros são:

$\mathrm{J}=0.362\left[\mathrm{Kgm}^{2}\right]$ - momento inercial do complexo canela-pé;

$\mathrm{m}=4.37[\mathrm{Kg}]$ - massa do complexo canela-pé;

$1=23.8[\mathrm{~cm}]$ - distância entre joelho e centro de massa do complexo canela-pé;

$\mathrm{B}=0.27[\mathrm{Nms} / \mathrm{rad}]$ - coeficiente de atrito viscoso;

$\lambda=41.208[\mathrm{Nm} / \mathrm{rad}]$ - coeficiente do termo exponencial;

$\mathrm{E}=2.024\left[\mathrm{rad}^{-1}\right]$ - coeficiente do termo exponencial; $\omega=2.918[\mathrm{rad}]$ - ângulo elástico de repouso do joelho;

$\tau=0.951[s]$ - coeficiente da função transferência;

$\mathrm{G}=42500[\mathrm{Nm} / \mathrm{s}]$ - coeficiente da função transferência;

$\mathrm{P}_{\mathrm{N}}$ - largura de pulso não referenciada;

$\mathrm{P}$ - largura de pulso;

Sendo $f_{21}\left(x_{1}(\mathrm{t})\right)$ indeterminada em $x_{1}=0$, o que dificulta a determinação das LMIs e das funções de pertinência do modelo fuzzy Takagi-Sugeno exato, expande-se $f_{21}\left(x_{1}(\mathrm{t})\right)$ em série de Taylor, solucionando o problema. Para o caso de $\theta_{v}=45^{\circ}$ expandiu-se até a $7^{\mathrm{a}}$ ordem. Em Gaino (2009), temse que $f_{21}\left(\mathrm{x}_{1}(\mathrm{t})\right)$ para $\theta_{v}=30^{\circ}$ expandiu-se a serie até a $5^{\mathrm{a}}$ ordem e para $\theta_{v}=60^{\circ}$ expandiu-se a serie até a $11^{\mathrm{a}}$ ordem.

Figura 4 - Série de Taylor de $f_{21}\left(x_{1}(\mathrm{t})\right) 7^{\circ}$ ordem.

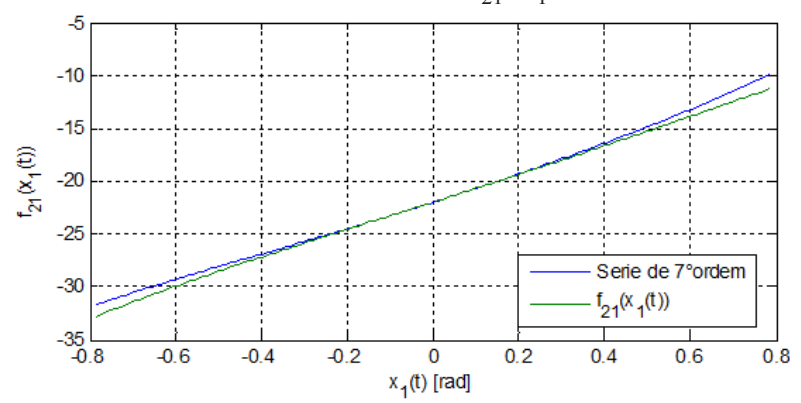

Fonte: Do Autor. 


\section{LMI aplicada em controle}

As LMIs são uma das mais importantes ferramentas dentro da teoria de controle (VAN ANTWERP; BRAATZ, 2000).

Algumas de suas características as tornaram especiais, como a facilidade de trabalho com diversos sistemas que dificilmente teriam soluções analíticas, uma grande variedade de especificações e restrições de projetos que podem ser expressas por LMIs e a utilização de algoritmos computacionais que otimizam o processo de solução dos problemas.

\section{Forma geral das desigualdades matriciais} lineares

Uma LMI em uma variável $x=\left[\begin{array}{llll}x_{1} & x_{2} & \ldots & x_{\mathrm{m}}\end{array}\right]^{\mathrm{T}} \in \mathbb{R}^{\mathrm{m}}$ é descrita da seguinte forma (BOYD et al., 1994):

$$
F(x)=x_{1} F_{1}+x_{2} F_{2}+\cdots+x_{m} f_{m} \leq F_{0}
$$

ou

$$
F(x)=\sum_{i=1}^{m} x_{i} F_{i}-F_{0} \leq 0
$$

sendo que $\mathrm{F}_{i}=\mathrm{F}_{i}^{\mathrm{T}} \in \mathbb{R}^{\mathrm{n} \times \mathrm{n}}, i=0, \ldots, \mathrm{m}$, são matrizes simétricas de ordem $n$, cujos autovalores são reais. A notação $\leq$ é usada no contexto usual para sinais de matrizes, isto é, $\mathrm{F}(\mathrm{x}) \leq 0$ é dita ser semidefinida negativa, sendo os autovalores de $\mathrm{F}(\mathrm{x})$ não$\operatorname{positivos}^{5}$.

Em outras palavras, a LMI F $(x)$ é uma funcional afim, mapeando um espaço vetorial na entrada, em um cone de matrizes simétricas semidefinidas negativas na saída. Portanto, uma propriedade inerente das LMIs é apresentar simetria em sua estrutura. Grosso modo, uma LMI pode ser vista como uma desigualdade com elementos matriciais e simétrica. Note que a desigualdade em (5) pode ser também estrita, isto é, $\mathrm{F}(\mathrm{x})<0$ ou $\mathrm{F}(\mathrm{x})>0$. Pode, ainda, ser do tipo $\mathrm{F}(\mathrm{x}) \geq 0$.

Uma propriedade importante das LMIs reside no fato de que seu conjunto solução, denotado por $\{x$ $\left.\in \mathbb{R}^{\mathrm{m}} \mid \mathrm{F}(x) \leq 0\right\}$, é convexo. Essa característica é fundamental, pois permite a utilização de algoritmos apropriados para resolver esse tipo de problema de forma bastante eficiente.

\section{Análise da estabilidade segundo Lyapunov}

Considere o sistema dinâmico linear autônomo da forma (AGUIRRE et al., 2007):

$$
\dot{x}=A x(t)
$$

sendo que $x \in \mathbb{R}^{\mathrm{n}}$ e, $\mathrm{A} \in \mathbb{R}^{\mathrm{n} \times \mathrm{n}}$.

Um ponto de equilíbrio para o sistema (7) é alcançado quando $\dot{x} \equiv 0$, isto é, quando anão há variação nas variáveis de estado. Todas as derivadas são nulas, significando que os estados não estão variando no tempo e, portanto, são indicados como pontos de equilíbrio.

O critério de estabilidade de Lyapunov é obtido definindo-se uma função quadrática escalar positiva:

$$
\begin{gathered}
V(x(t))=x^{T}(t) P x(t)>0, \text { sendo } P=P^{T}>0, \\
P \in \mathbb{R}^{n \times n}
\end{gathered}
$$

e avaliando a derivada ao longo das trajetórias de $x(\mathrm{t})$, isto é:

$$
\dot{V}(x(t))=x^{T}(t)\left(A^{T} P+P A\right) x(t)
$$

Como $\mathrm{V}(x(\mathrm{t}))$ é definida positiva, para se ter estabilidade assintótica $(x(\mathrm{t}) \equiv 0$ é o estado de equilíbrio) é necessário que $\dot{V}(x)<0$, ou

$$
A^{T} P+P A<0, \operatorname{com} P>0
$$

\footnotetext{
5 Adota-se notação semelhante para: matriz semidefinida positiva $(\mathrm{F} \geq 0)$ sendo todos os seus autovalores não-negativos; matriz definida positiva $(\mathrm{F}>0)$ sendo todos os seus autovalores positivos; matriz definida negativa $(\mathrm{F}<0)$ tendo todos os seus autovalores negativos.
} 
Portanto o Teorema de Lyapunov garante que o sistema (7) é assintoticamente estável se e somente se existir uma matriz $\mathrm{P}=\mathrm{P}^{\mathrm{T}}>0$ satisfazendo a LMI (10).

\section{Controle utilizando LMI}

Considere a planta linear, invariante no tempo, controlável e observável (OGATA, 2004):

$$
\begin{gathered}
\dot{x}=A x+B u \\
y=C x
\end{gathered}
$$

sendo $x \in \mathbb{R}^{\mathrm{n}}$ o vetor de estado, $u \in \mathbb{R}^{\mathrm{m}}$ a entrada de controle, $y \in \mathbb{R}^{\mathrm{m}}$ a saída do sistema, $A \in R n \times \mathrm{n}$ a matriz característica do sistema, $\mathrm{B} \in \mathbb{R}^{\mathrm{n} \times \mathrm{m}}$ a matriz de entrada do sistema e $C \in \mathrm{R}^{\mathrm{m} \times \mathrm{n}}$ a matriz de saída do sistema (COVACIC, 2006). Desde que este sistema seja controlável é possível especificar uma matriz K, denominada matriz ganho de retroação de estados, satisfazendo a lei de controle:

$$
u=-K x
$$

que tornam negativa a parte real dos pólos do sistema em malha fechada, satisfazendo assim o requisito da estabilidade.

Seguindo a análise da estabilidade segundo Lyapunov tem-se as seguintes desigualdades, $\mathrm{P}=\mathrm{P}^{\mathrm{T}}$.

$$
\begin{gathered}
(A-B K)^{T} P+P(A-B K)<0 \\
P>0
\end{gathered}
$$

Observe que (13) não é uma LMI, pois apresenta um produto envolvendo duas variáveis. Multiplicando cada termo em (13) por $\mathrm{P}^{-1}$ e mantendo a propriedade de não unicidade das LMIs, tem-se:

$$
A P^{-1}-B K P^{-1}+P^{-1} A^{T}-P^{-1} K^{T} B^{T}<0
$$

definindo $\mathrm{X}=\mathrm{P}^{-1}$ e $\mathrm{G}=\mathrm{KP}^{-1}=\mathrm{KX}$, tem-se enfim a LMI resultante (GAINO, 2009):

$$
\begin{gathered}
A X-B G+X A^{T}-G^{T} B^{T}<0 \\
X>0
\end{gathered}
$$

A matriz de ganho de retroação de estados é obtida por:

$$
K=G X^{-1}
$$

\section{Controle Fuzzy Takagi-Sugeno}

Um modelo fuzzy Takagi-Sugeno descreve de maneira aproximada um sistema (global) não linear, através da combinação de $n$ modelos locais lineares invariantes no tempo, considerando-se uma lei de controle de realimantação linear para cada modelo linear local. Obtêm-se modelos locais lineares através de valores máximos e mínimos da função, combinando-os de maneira convexa.

\section{Modelos Locais Lineares}

Definindo os seguintes modelos (TAKAGI; SUGENO, 1985), (TANAKA; WANG, 2001):

$$
\begin{gathered}
\dot{x}=A_{i} x(t)+B_{i} u(t) \\
y(t)=C_{i} x(t)
\end{gathered}
$$

para $i=1,2, \ldots, p$, considere as funções de pertinência:

$$
\omega^{i}(z(t))=\prod_{i=1}^{p} \mu_{j}^{i}\left(z_{j}(t)\right)
$$

sendo $\omega^{i}(\mathrm{z}(\mathrm{t})) \geq 0$ tais que $\sum_{i=1}^{r} \omega^{i}(z(t))>0$.

Para trabalhar com modelos fuzzy Takagi-Sugeno adota-se $x(\mathrm{t})=\mathrm{z}(\mathrm{t})$, sendo $x(\mathrm{t})$ o vetor de estado do sistema não linear. E aplicando média ponderada acha-se:

$$
\begin{gathered}
\dot{x}(t)=\frac{\sum_{i=1}^{r} \omega^{i}(x(t))\left(A_{i} x(t)+B_{i} u(t)\right)}{\sum_{i=1}^{r} \omega^{i}(x(t))} \\
=A(\alpha(x(t))) x(t)+B(\alpha(x(t))) u(t) \\
=A(\alpha) x(t)+B(\alpha) u(t) \\
\therefore \alpha(x(t))=\frac{\omega^{i}(x(t))}{\sum_{i=1}^{r} \omega^{i}(x(t))} \\
y(t)=C(\alpha) x(t)
\end{gathered}
$$

sendo: $\alpha(x(\mathrm{t})) \geq 0$ e $\sum_{i=1}^{r} \alpha(x(t))=1$.

Analogamente e utilizando o conceito de Compensação Distribuída Paralela (CDP), com leis de controle baseadas em controle linear, foi projetado um compensador para cada regra do modelo fuzzy: 


$$
u(t)=-F(\alpha) x(t)
$$

Substituindo (22) em (19):

$\dot{x}(t)=\sum_{i=1}^{r} \sum_{j=1}^{r} \alpha_{i}(x(t)) \alpha_{j}(x(t))\left(A_{i}-B_{i} F_{j}\right) x(t)$

sendo $r$ o numero de modelos locais.

Aplicando o conceito de estabilidade tem-se:

$$
\dot{V}(x(t))=\dot{x}(t)^{T} P x(t)+x(t)^{T} P \dot{x}(t)<0
$$

Em malha aberta o resultado é:

$$
\dot{V}(x(t))=x(t)^{T} \sum_{i=1}^{r} \alpha_{i}(x(t))\left(A_{i}^{T} P+P A_{i}\right) x(t)<0
$$

Considerando:

$$
G_{i j}=A_{i}-B_{i} F_{j}
$$

é obtida a seguinte lei de controle em malha fechada (AVELLAR, 2001):

$$
\begin{gathered}
P>0, \quad P=P^{T} \\
G_{i i}^{T} P+P G_{i i}<0, \quad \forall i \\
\left(\frac{G_{i j}^{T}+G_{i j}^{T}}{2}\right) P+P\left(\frac{G_{i j}+G_{i j}}{2}\right) \leq 0, \quad i<j
\end{gathered}
$$

\section{Controle por LMI}

Partindo do sistema (19), (TANAKA et al.,1998), (TANAKA; WANG, 2001), realimentado pela lei de controle (22) e das condições de controle descritas anteriormente, reescrevem-se as condições de controle, adotando (26), $\mathrm{X}=\mathrm{P}^{-1}$ e $\mathrm{M}_{i}=\mathrm{F}_{i} \mathrm{X} \rightarrow \mathrm{F}_{i}=\mathrm{M}_{i} \mathrm{X}^{-1}$.

$$
\begin{gathered}
X=X^{T}>0 \\
-X A_{i}^{T}+M_{i}^{T} B_{i}^{T}-A_{i} X+B_{i} M_{i}>0 \\
-X A_{i}^{T}+M_{j}^{T} B_{i}^{T}-X A_{j}^{T}+M_{i}^{T} B_{j}^{T}-A_{i} X+B_{i} M_{j}- \\
A_{j} X+B_{j} M_{i} \geq 0
\end{gathered}
$$

Deste modo, têm-se as LMIs que garantem a estabilidade do sistema.

\section{Taxa de Decaimento}

A velocidade de resposta está relacionada com a taxa de decaimento $(\beta)$. Para obter uma taxa de decaimento de $\beta$, sendo $\beta>0$, faz-se sendo esta condição satisfeita em toda trajetória de $x(\mathrm{t})$ do sistema, (TANAKA et al., 1998), (TANAKA; WANG, 2001), (BOYD et al., 1994)). Ou seja, podese garantir um tempo de estabilização, limitando o tempo de transitório do sistema realimentado.

Incluindo a especificação da taxa de decaimento nas equações de controle descritas em (30)-(32), tem-se:

$$
\begin{gathered}
X=X^{T}>0 \\
-X A_{i}^{T}+M_{i}^{T} B_{i}^{T}-A_{i} X+B_{i} M_{i}-2 \beta P>0 \\
-X A_{i}^{T}+M_{j}^{T} B_{i}^{T}-X A_{j}^{T}+M_{i}^{T} B_{j}^{T}-A_{i} X+B_{i} M_{j}-A_{j} X+ \\
B_{j} M_{i}-4 \beta P \geq 0
\end{gathered}
$$

\section{Restrição de Entrada}

Assumindo que a condição inicial é conhecida $(x(0))$, a restrição $\|u(\mathrm{t})\| \leq \mu$ é garantida para todo tempo $\mathrm{t} \geq 0$, se as LMIs (TANAKA; WANG, 2001):

$$
\begin{gathered}
{\left[\begin{array}{cc}
1 & x(0)^{T} \\
x(0) & X
\end{array}\right] \geq 0} \\
{\left[\begin{array}{cc}
X & M_{i}^{T} \\
M_{i} & \mu^{2} I
\end{array}\right] \geq 0}
\end{gathered}
$$

sendo $\mathrm{X}=\mathrm{P}^{-1}$ e $\mathrm{M}_{i}=\mathrm{F}_{i} \mathrm{X}$, forem satisfeitas simultaneamente com as LMIs (30), (31) e (32) ou (33), (34) e (35) caso haja uma taxa de decaimento desejada.

\section{Resultados e Discussões}

Considerando o ângulo de estabilização $\theta_{v}=45^{\circ}$, obtém-se o torque ativo e a amplitude do pulso de entrada, por (3) e (4):

$$
\begin{gathered}
M_{a 0}=7.0107[\mathrm{Nm}] \\
P_{0}=1.6496 \cdot 10^{-4}[\mathrm{~s}]
\end{gathered}
$$


Para outros ângulos os valores de $\mathrm{M}_{\mathrm{a} 0}$ e $\mathrm{P}_{0}$ são diferentes, mas a obtenção do controle dáse analogamente. Em ângulos maiores que $45^{\circ}$, é necessário expandir $f_{21}\left(x_{1}(\mathrm{t})\right)$ em série de Taylor de ordem mais elevada, com o intuito de diminuir o erro dessa linearização.

\section{Controle exclusivo por LMI}

No controle do sistema de malha fechada da equação (1) com seus respectivos valores determinados, usam-se as LMIs apresentadas em (15) para encontrar a matriz de ganho de retroação de estados:

$$
K=10^{-3} \cdot\left[\begin{array}{lll}
-0.1191 & 0.0167 & 0.0040
\end{array}\right]
$$

Substitui-se $\mathrm{P}_{\mathrm{n}}=u=-\mathrm{K} x$ em (1) forma-se os seguintes sistemas:

$$
\begin{gathered}
\dot{x}_{1}(t)=x_{2}(t) \\
\dot{x}_{2}(t)=\tilde{f}_{21}(0) x_{1}(t)-\frac{B}{J} x_{2}(t)+\frac{1}{J} x_{3}(t) \\
\dot{x}_{3}(t)=-\frac{1}{\tau} x_{3}(t)-\frac{G}{\tau}\left[K(1) x_{1}(t)+K(2) x_{2}(t)+\right. \\
\left.K(3) x_{3}(t)\right]
\end{gathered}
$$

sendo $K=[K(1) K(2) K(3)]$.

Fazendo uso do MATLAB juntamente com a função ode23t são obtidos $x_{1}(\mathrm{t}), x_{2}(\mathrm{t})$ e $x_{3}(\mathrm{t})$, conforme os gráficos das Figuras 5 a 7 .

A largura de pulso $P(t)$ se dá pela soma de $P_{n}$ com $P_{0}$, como:

$$
P_{n}=-K(1) x_{1}(t)+K(2) x_{2}(t)+K(3) x_{3}(t)
$$

resultando na lei de controle mostrada na Figura 8.

Figura 5 - Posição exercida pela perna.

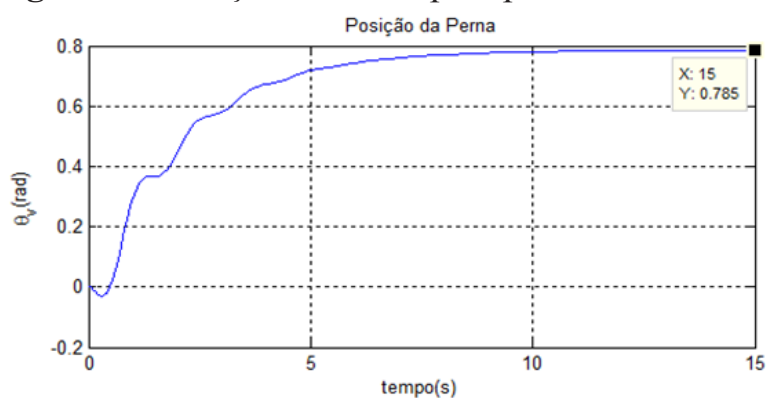

Fonte: Dados do autor.
Figura 6 - Velocidade angular exercida pela perna.

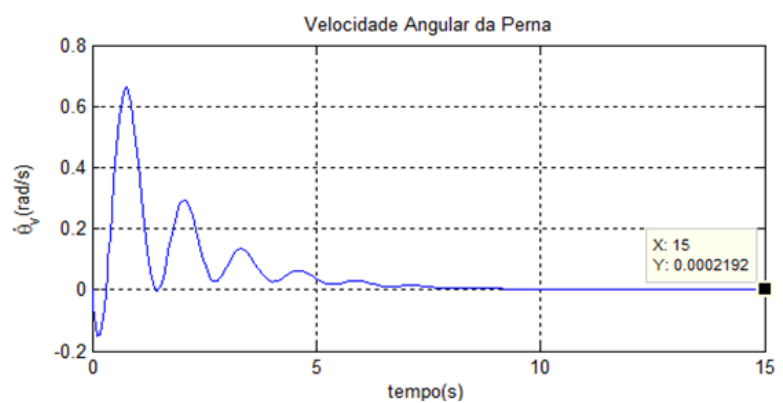

Fonte: Dados do autor.

Figura 7 - Torque ativo exercido pela perna.

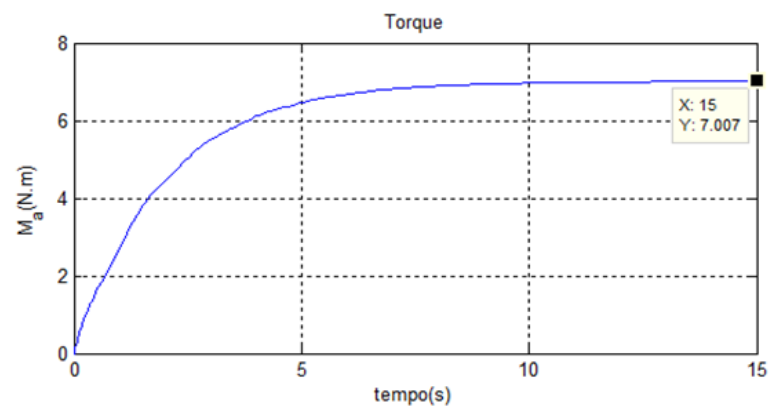

Fonte: Dados do autor.

Figura 8 - Largura de pulso aplicada.

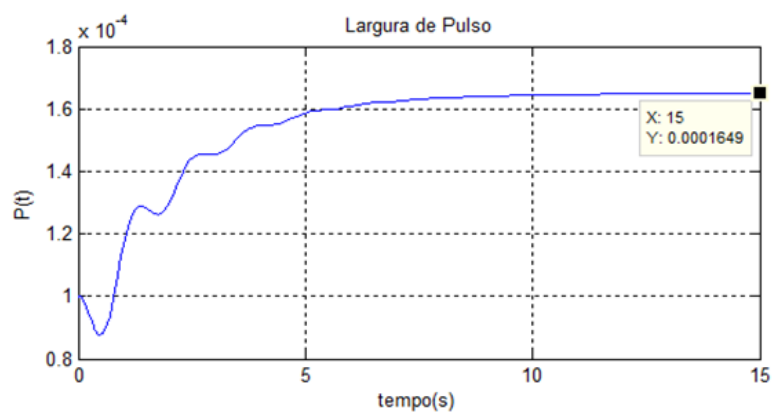

Fonte: Dados do autor.

\section{Controle por Fuzzy Takagi-Sugeno}

Este controle foi realizado com o auxílio do MATLAB. Analisando a curva não linear aproximada de $f_{21}\left(x_{1}(\mathrm{t})\right)$ e sendo $0<\theta v<45^{\circ}$, temse os valores de máximo e mínimo da função:

$$
\begin{aligned}
& f_{21 \text { Max }}=-11,24 \\
& f_{21 \text { min }}=-21,99
\end{aligned}
$$


Assim, trabalhando com dois modelos locais $\left(\theta_{v}=0, \theta_{v}=45^{\circ}\right)$, tem-se $\mathrm{r}=2$. Definindo as funções de pertinência usadas para a solução do sistema:

$$
f_{21}\left(x_{1}(t)\right)=f_{21 \text { Max }} \alpha_{1}\left(x_{1}(t)\right)+f_{21 \min } \alpha_{2}\left(x_{1}(t)\right)
$$

sendo: $\left(x_{1}(t)\right) \geq 0$ e $\sum_{i=1}^{r} \alpha\left(x_{1}(t)\right)=1$.

Utiliza-se a seguinte função de pertinência estudada em (PIETROBOM, 1999), para a modelagem do sistema:

$$
\alpha_{1}=\left(1-\frac{1}{1+e^{\left(-14 \cdot\left(x(1)-\frac{\pi}{8}\right)\right)}}\right) \cdot\left(\frac{1}{\alpha_{2}=1-\alpha_{1}}\right)
$$

Representada por:

Figura 9 - Função de Pertinência para $x_{1} \in[0, \pi / 4]$.

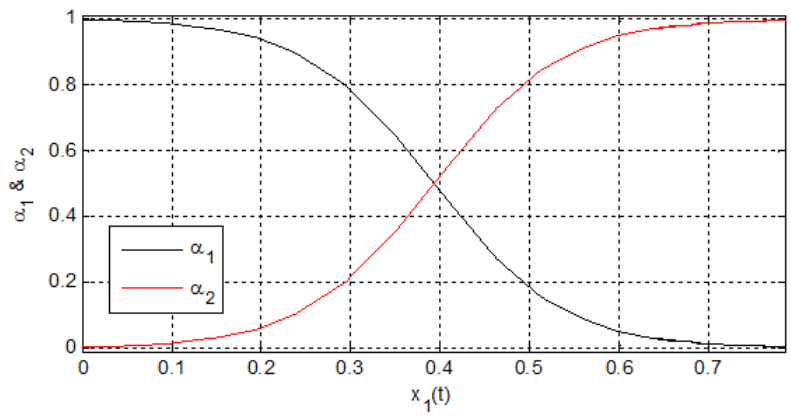

Fonte: Dados do autor.

Para a modelagem fuzzy T-S, dada em (19) e (20), regida pela lei de controle (30), (31) e (32), factíveis ${ }^{6}$. Resolvendo as LMIs tem-se o ganho do controlador dado por:

$$
\begin{gathered}
F_{1}=10^{-3} \cdot\left[\begin{array}{lll}
0.6051 & 0.3440 & 0.0793
\end{array}\right] \\
F_{2}=10^{-3} \cdot\left[\begin{array}{lll}
0.7379 & 0.3928 & 0.0942
\end{array}\right]
\end{gathered}
$$

As Figuras 10 a 13 mostram a resposta do sistema com o controle fuzzy T-S. Lembrando-se que:

$$
\begin{gathered}
x_{1}=\Delta \theta_{v}=\theta_{v}-\theta_{v 0} \rightarrow \theta_{v}=x_{1}+\theta_{v 0} \\
x_{2}=\dot{x}_{1}=\Delta \dot{\theta}_{v} \\
x_{3}=\Delta M_{a}=M_{a}-M_{a 0} \rightarrow M_{a}=x_{3}+M_{a 0} \\
u(t)=P_{n}=\mathrm{P}-\frac{M_{a 0}}{G}=P-P_{0} \rightarrow P=u(t)+P_{0}
\end{gathered}
$$

sendo o ponto de operação do sistema $\left(\theta_{v}, \dot{\theta}_{v}, M_{a}\right)=\left(\theta_{v}, 0, M_{a 0}\right)$ , tem-se que o sistema estabiliza-se em:

$$
\begin{gathered}
\theta_{v}=\pi / 4=0.7854[\mathrm{rads}] \\
\dot{\theta}_{v}=0[\mathrm{rad} / \mathrm{s}] \\
M_{a}=M_{a 0}=7,0107[\mathrm{Nm}] \\
P=P_{0}=1.6496 \cdot 10^{-4}[\mathrm{~s}]
\end{gathered}
$$

Figura 10 - Posição da perna modelada por fuzzy T-S.

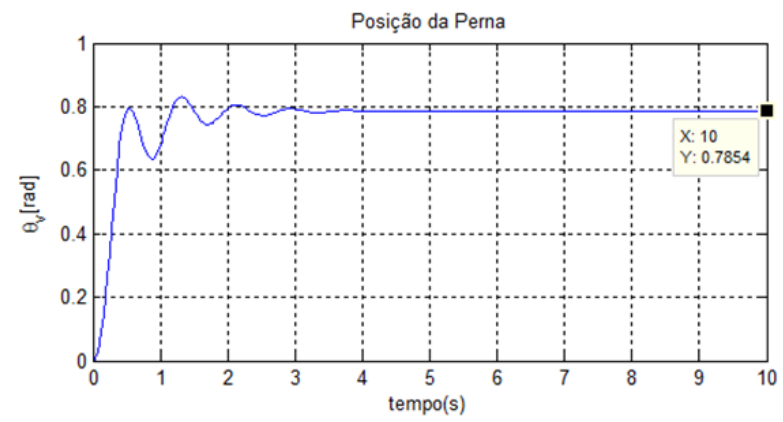

Fonte: Dados do autor.

Figura 11 - Velocidade angular modelada por fuzzy T-S.

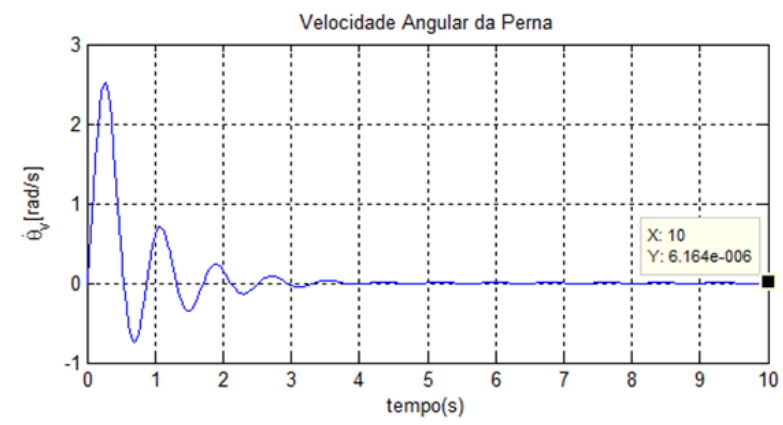

Fonte: Dados do autor.

\footnotetext{
${ }^{6}$ Uma solução factível para as LMIs garante a existência de matrizes dependentes de parâmetro usados em Lyapunov, que asseguram a estabilidade robusta, permitindo-se projetar uma matriz de realimentação de estados que estabiliza o sistema.
} 
Figura 12 - Torque ativo modelado por fuzzy T-S.

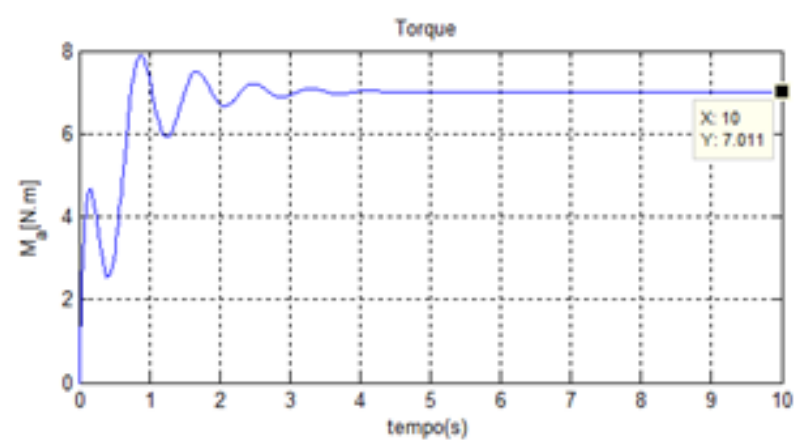

Fonte: Dados do autor.

Figura 13 - Largura de pulso aplicada.

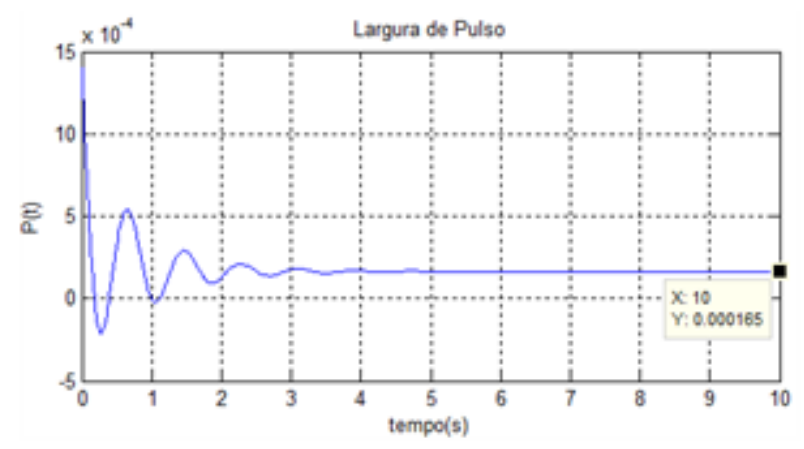

Fonte: Dados do autor.

Como se pode observar na Figura 13, a largura de pulso a ser aplicada ao sistema atingiu valores negativos, o que é inviável no sistema considerado. Desta forma, foi necessário incluir as LMIs (36) e (37), com $\mu^{2}=15 \cdot 10^{-4}$, de modo a garantir uma largura de pulso sempre positiva, como se vê na Figura 17. Como $P_{n}=P-P 0$, isto é, $P=P_{n}+P_{0}$, uma condição suficiente para garantir que $\mathrm{P}>0\left(\mathrm{P}_{\mathrm{n}}>-\mathrm{P}\right)$ é determinar que $\left|P_{\mathrm{n}}\right|<P_{0}$. Com isto, resolvendo as LMIs tem-se o ganho do controlador dado por:
Figura 14 - Posição da perna modelada por fuzzy T-S com restrição na entrada.

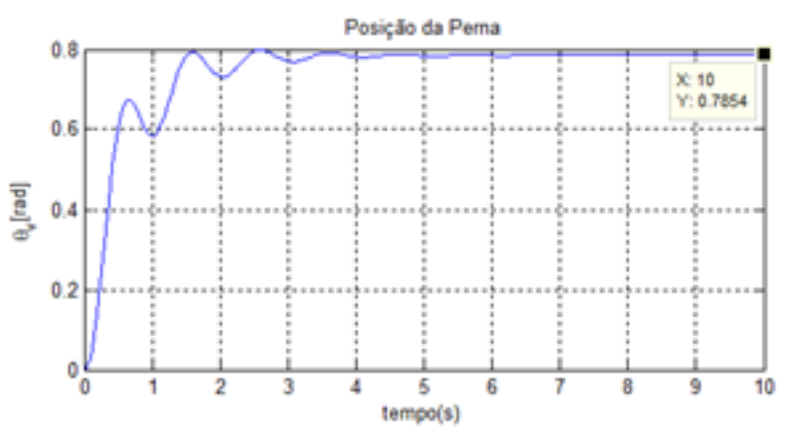

Fonte: Dados do autor.

Figura 15 - Velocidade angular modelada por fuzzy T-S, com restrição na entrada.

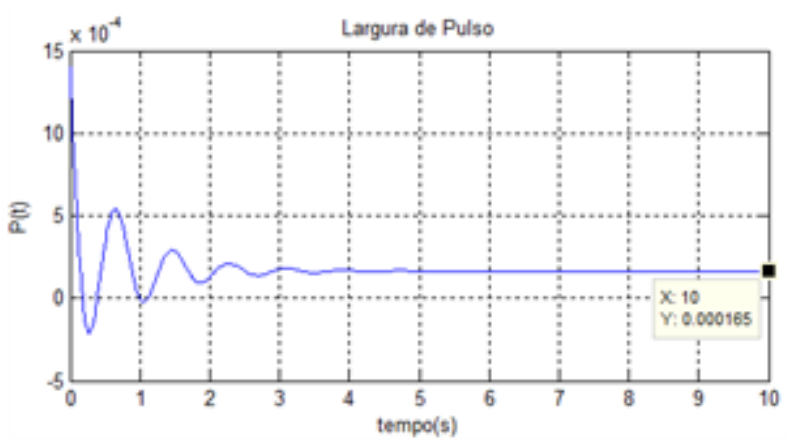

Fonte: Dados do autor.

Figura 16 - Torque ativo modelado por fuzzy T-S, com restrição na entrada.

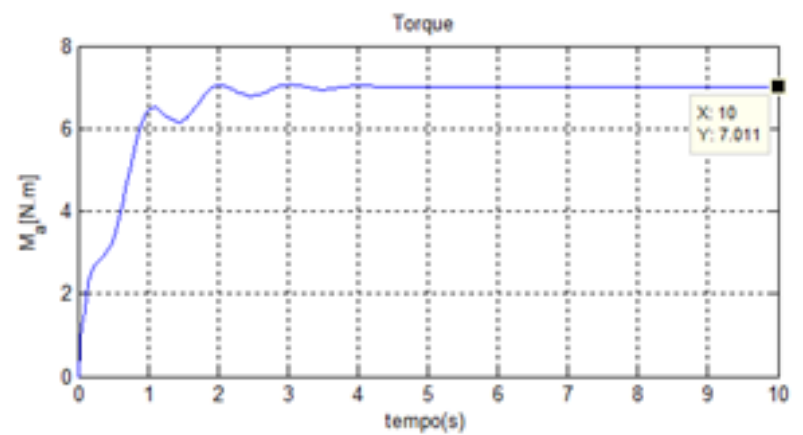

Fonte: Dados do autor. 
Figura 17 - Largura de pulso aplicada.

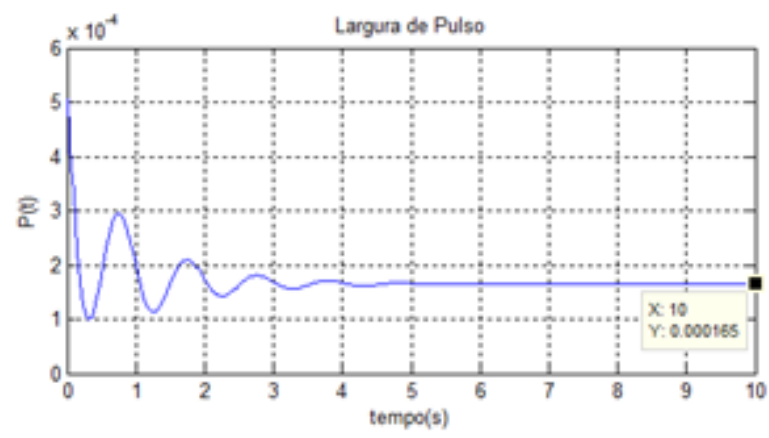

Fonte: Dados do autor.

A utilização de Modelos fuzzy Takagi-Sugeno, na modelagem de sistemas não lineares apresenta uma aproximação mais exata desse sistema, visto que o regulador global é não linear, formado por uma combinação fuzzy de reguladores lineares individuais. Já o controle por LMIs apresenta uma aproximação mais grotesca, mas contém resultados condizentes com um controle efetivo.

Na prática, já existe esse sistema implementado em malha aberta na UNESP, em Ilha Solteira. Assim, é necessária a implementação de um sistema realimentado, utilizando como sensor o eletrogoniômetro. Este trabalho apresenta o sistema de malha fechada que deve ser implementado em microcontroladores ou com componentes físicos. Caso ocorra um carregamento externo, o ângulo se manterá constante, visto que a realimentação busca o erro nulo dado um ângulo de referência, deste modo variando a largura do pulso, variando, consequentemente o torque ativo e a aceleração o que garantem o ângulo desejado.

\section{Conclusões}

O projeto de controle da posição da perna de pacientes paraplégicos com eletroestimulação aplicada ao músculo quadríceps obteve resultados interessantes. Com base nas desigualdades matriciais lineares (LMIs), utilizando modelos
Fuzzy Takagi-Sugeno (T-S), mostrou-se uma excelente forma de controle, podendo-se ajustar o tempo de estabilização da forma desejada. No projeto, devem ser levados em conta a velocidade angular e o torque gerado na perna, que podem ser indesejáveis em determinados pontos, como no caso de um tempo de estabilização muito baixo, o que torna a resposta muito abrupta.

As simulações no MATLAB comprovam a eficiência do método proposto, com o ângulo da articulação do joelho variando no intervalo de $0^{\circ}$ a $45^{\circ}$, sendo o sistema de controle projetado, considerando todas as não linearidades da planta e satisfazendo às restrições de projeto (estabilidade segundo Lyapunov, taxa de decaimento e restrição ao sinal de entrada), mostrando-se um método completo e com todos os aspectos bem definidos. Há a possibilidade de obter-se a estabilidade para qualquer ângulo, alterando, assim, os cálculos.

Os próximos passos deste trabalho são a utilização do Controle com Estrutura Variável e a otimização da função de pertinência para modelos fuzzy, a fim de obter-se o controle do sistema mesmo com variação de certos parâmetros e distúrbios na entrada do sistema, bem como a melhor adequação dos modelos fuzzy Takagi-Sugeno.

\section{Referências}

AGUIRRE, L. A.; SILVA, A. P. A.; CAMPOS, M. F. M.; AMARAL, W. C. (Org.) Enciclopédia de Automática: controle e automação. São Paulo: Blucher, 2007. v. 1.

AVELLAR, R. G. Controle de sistemas não lineares baseados em LMI utilizando modelos fuzzy. Ilha Solteira: FEIS/UNESP, 2001, Estudos Especiais, Universidade Estadual Paulista "Júlio de Mesquita Filho", Ilha Solteira, 2001.

BIAZETO, A. R. Contribuições de identificação de sistemas lineares a não-lineares para o caso do paciente paraplégico. 2011. Trabalho de Conclusão de Curso (Graduando em 2011) - Universidade Estadual de Londrina, Londrina.

BOYD, S.; GHAOUI, L. E.; FERON, E.; BALAKRISHNAN, V. (Org.). Linear matrix inequalities in system and control theory. Philadelphia: SIAM, 1994. 
CHEN, Y. L.; CHANG, W. H.; WONG, M. K.; TANG, F. T.; CHEN, H. I.; HUANG, C. S.; KUO, T. S. A treadmill apparatus with a computer-assisted functional electrical stimulation system by foot switch controlled for restaration af am bulation in stroke patients. In: INTERNATIONAL CONFERENCE IEEE/EMBS, 19., 1997, Chicago. Proceedins... Chicago: IEEE, 1997.

COVACIC, M. R.; TEIXEIRA, M. C. M.; ASSUNÇÃO, E.; GAINO, R. LMI-based algorithm for strictly positive real systems with static output feedback. Systems \& Control Letters, Amsterdam, v. 61, p. 521-527, 2012.

COVACIC, M. Sintese de sistemas ERP baseada em LMIS e controle com estrutura variável. 2006. Tese (Doutorado em 2006) - Universidade Estadual Paulista, Ilha Solteira.

FERRARIN, M.; PEDOTTI, A. The relationship between electrical stimulus and joint torque: a dynamic model. IEEE Transactions on Rehabilitation Engineering, San Diego, v. 8, n. 3, p. 342-352, 2000.

GAINO, R. Controle de movimentos de pacientes paraplégicos utilizando modelos Fuzzy T-S. 2009, Tese (Doutorado em 2009) - Programa de Pós-Graduação em Engenharia Elétrica, Universidade Estadual Paulista "Júlio de Mesquita Filho", Ilha Solteira.

GAINO, R.; SILVA, T. I.; SANCHES, M.A.A.; FREITAS, R. L. B.; SATO, F. H.; SANTOS, E. A.; CARVALHO, A. A.; TEIXEIRA, M. C. M.; ASSUNÇÃO, E.; MACHADO, E. R. M. D. Controle digital para FES no estímulo do músculo quadríceps em pacientes paraplégicos. In: BRAZILIAN CONFERENCE ON DYNAMICS, CONTROL AND THEIR APPLICATIONS, 6., 2007, São José do Rio Preto. Anais... São José do Rio Preto: IBILCE-UNESP, 2007a. p. 764-771.

GAINO, R.; TEIXEIRA, M. C. M.; CARDIM, R.; CARVALHO A. A.; ASSUNÇÃO, E.; SANCHES, M. A. A. Controle não-linear Fuzzy Takagi-Sugeno do movimento de paraplégicos utilizando acelerômetros. In: CONGRESSO BRASILEIRO DE ENGENHARIA BIOMÉDICA, 21., 2008. Anais... Salvador: CBEB, 2008. p. 1254-1257.

GAINO, R.; TEIXEIRA, M. C. M.; CARVALHO, A. A.; ASSUNÇÃO, E.; SILVA, T. I. Reguladores e observadores fuzzy takagi-sugeno para variar o Ângulo da articulação do joelho de um paciente paraplégico. In: SIMPÓSIO BRASILEIRO DE AUTOMAÇÃO INTELIGENTE, 7., 2007, Florianópolis. Anais... Florianópolis: SBAI, $2007 \mathrm{~b}$. p. 1-6.

GAINO, R.; TEIXEIRA, M. C. M.; CARVALHO A. A.; ASSUNÇÃO, E.; CARDIM, R.; SANCHES, M.

A. A.; COVACIC, M. R. Realimentação derivativa e modelos fuzzy Takagi-Sugeno para controle da articulação do joelho de pacientes paraplégicos com uso de acelerômetros. Revista Brasileira de Engenharia Biomédica, Rio de Janeiro, v. 27, n. 2, p. 67-78, 2011.

HANDA, T.; TAKAHASHI, H.; SAITO, C.; HANDA, Y.; ICHIE, M.; KAMEYAMA, J.; HOSHIMIYA, N. Development of an FES system controlled by emg signals. Annual International Conference of the IEEE Engineerring in Medicine and Biology Society, New York, v. 12, n. 5, 1990.

OGATA, K. Modern control engineering. Upper Saddle River: Prentice Hall, 2004.

PIETROBOM, H. C. Controle de sistemas não-lineares baseados em lmi utilizando modelos fuzzy. 1999. 94 p. Dissertação (Mestrado em Engenharia Elétrica) - Faculdade de Engenharia, Universidade Estadual Paulista, Ilha Solteira.

PRADO, T. A.; GAINO, R.; CARVALHO A. A.; PEREIRA, M. C.; BATISTA, E. A.; CARNEIRO, A. M. Desenvolvimento de uma ferramenta para projeto de controladores PID aplicados em estimulação neuromuscular funcional. In: CONGRESSO BRASILEIRO DE ENGENHARIA BIOMÉDICA, 21, 2008, Salvador. Anais... Salvador: CBEB, 2008. p. 891894.

ROBINSON, A. J. Eletrofisiologia Clínica: eletroterapia, e teste fisiológico. 2. ed. Porto Alegre: Armed, 2001.

SILVA, T. I. Implementação de um sistema para geração e avaliação de movimentos em pacientes hemiplégicos. 2007. Tese (Doutorado em 2007) - Programa de PósGraduação em Engenharia Elétrica, Universidade Estadual Paulista "Júlio de Mesquita Filho", Ilha Solteira.

TAKAGI T.; SUGENO M. Fuzzy identification of system and its applications to modeling and control. IEEE Transactions on Systems, Man, and Cybernetics, New York, v. 15, n. 1, p. 116-132, 1985.

TANAKA, K.; IKEDA, T.; WANG, O. H. Fuzzy regulators and fuzzy observers: relaxed stability conditions and LMI-based designs. IEEE Transactions on Fuzzy Systems, New York, v. 6, n. 2, p. 250-265, 1998.

TANAKA, K.; WANG, H. O. Fuzzy control systems design and analysis. Canada: Wiley-Interscience, 2001.

TANIGUCHI, T.; TANAKA, K.; OHATAKE, H.; WANG, H. O. Model construction, rulereduction, and robust compensation for generalized form of TakagiSugeno fuzzy systems. IEEE Transactions on Fuzzy Systems, New York, v. 9, n. 4, p. 525-537, 2001. 
TEIXEIRA, M. C. M.; ASSUNÇÃO, E.; AVELLAR, R. G. On relaxed LMI-based designs forfuzzy regulators and fuzzy observers. IEEE Transactions on Fuzzy Systems, New York, v. 11, p. 613-623, 2003.

TEIXEIRA, M. C. M.; DEAECTO, G. S.; GAINO, R.; ASSUNÇÃO, E.; CARVALHO, A. A.; FARIAS, U. C. Projeto de um controlador fuzzy Takagi-Sugeno para variar o ângulo da articulação do joelho. In: CONGRESSO BRASILEIRO DE AUTOMÁTICA, 16. 2006, Salvador. Anais... Salvador: CBA, 2006. p. 22872292.

TEIXEIRA, M. C. M.; ZAK, S. H. Stabilizing controller design for uncertain non-linear systems using fuzzy models. IEEE Transactions on Fuzzy Systems, New York, v. 7, n. 2, p. 133-142, 1999.

VAN ANTWERP, J. G.; BRAATZ, R. D. A tutorial on linear and bilinear matrix inequalities. Journal of Process Control, Oxford, v. 10, p. 363-385, 2000.

YU, W.; MARUSIHI, M.; YOKOI, H.; MANO, Y.; KAKAZU, Y. An architecture for adaptive functional electrical stimulation for hemiplegic lower limb activities, Proceedings of the 1999. IEEE International Workshop on Robot and Human Interaction, Pisa, Sept. 1999.

Recebido em 12 Julho 2011- Received on July 12, 2011. Aceito em 31 Julho, 2012 - Accepted on July 31, 2012. 
